\title{
Exploration of Evaluation Index for Obstetric Anesthesiologists Training in China Using Modified Delphi Method, Experiences From Pilot Project of PKUHSC
}

\author{
Xiaoqing Zhang \\ Peking University Third Hospital \\ Yang Zhou \\ Peking University Third Hospital \\ Hong Zeng \\ Peking University Third Hospital \\ Min Li \\ Peking University Third Hospital \\ Jun Wang \\ Peking University Third Hospital \\ Changyi Wu \\ Peking University Third Hospital

\section{Xiangyang Guo} \\ Peking University Third Hospital \\ Mao Xu ( $\nabla$ xmputh@163.com) \\ Haidian Dist
}

\section{Research Article}

Keywords: evaluation index, obstetric anesthesiologists, subspecialty training, Delphi method

Posted Date: September 9th, 2021

DOI: https://doi.org/10.21203/rs.3.rs-882280/v1

License: (9) This work is licensed under a Creative Commons Attribution 4.0 International License. Read Full License 


\section{Abstract}

\section{Background}

With the gruadual liberation of fertility policy and the increased number of pregnant women at high-risk in China, a large number of obstetric anesthesiologists (OA) with competency were urgently demanded. In order to establish a replicable and standardized training system for OA specialists, Peking University Health Science Center, one of the first ratified specialists training centers in China, initiated this explorative project. As this is a continuous improving process, the final evaluation index has not been formulated.

Method

A working group was set up to devise survey instrument and the initial index draft. We initiated a nationwide questionnaire pooling opinions of 40 experts from Youth committee of China Anesthesiology Association to construct the evaluation index based on modified Delphi method to reach a consensus. Experts' demographic information, enthusiasm, authority, and agreement consensus were surgeyed. The final evaluation index would be constructed based on experts' opinion.

Results

Data were collected and analyzed for stability of the experts' enthusiasm ( $97.5 \%$ and $87.2 \%$, respectively), authority ( $0.91 \pm 0.07$ and $0.90 \pm 0.13$ for the respective two rounds), and agreement consensus ( $16.7 \%$ and $1.3 \%$ respectively; Kendal/ coefficient were $0.26 p=0.415$ and $0.147 p=0.000$, respectively). After two rounds of the survey, the evaluation standard was achieved with 2 items deleted, 5 items added, and 11 major revisions according to experts' opinion. Revisions mainly focused on clinical practical ability $(72 \%)$ and the research ability $(28 \%)$.

\section{Conclusion}

The final evaluation index was constructed consisting 3 categories (practice ability, non-technical skills, and teaching and research capabilities) with 5 primary and 14 secondary evaluation standards which further divided into 51 items. We hope it could be widely adopted as a standardized evaluation reference for obstetric anesthesiologists training.

\section{Background}

Maternal health has been incorporated into the United Nations Millennium Development Goals and the United Nations Sustainable Development Goals [1, 2]. Data from United States showed that severe morbidity was highest in women aged $>40$ years. [3, 4]. With the comprehensive liberalization of fertility policy in China, the number of more older pregnant women and pregnant women at high-risk increased significantly $[5,6]$. A cross-sectional study demonstrated that $81.12 \%$ hospitals lacked neuraxial labor analgesia service due to the shortage of anesthesiologists, which contributed to the high percentage of 
cesarean section rate $[7,8]$. The the incidence of uterus scarring complicated with placenta previa, uterine rupture, and postpartum hemorrhage was elevated, challenging medical institutions and demanding sufficient number of obstetric anesthesiologists $(O A)$ with competency $[9,10]$. However, the institutions providing obstetrical anesthesia in China are greatly understaffed, and the quality of obstetric anesthesia care from different regions vary significantly. There is no subspecialty-trained OAs in China, currently. In order to satisfy the demand for competent OAs and provide high-quality obstetric anesthesia, it is important to establish the subspecialty training system with standardized evaluation index.

In December 2015, the former National Health and Family Planning Commission, the Medical Reform Office of the State Council and other eight ministries and commissions jointly issued the guidance on the pilot project of establishment of a standardized training system for specialists in China; In June 2017, three pilot specialty training programs were launched and the medical education framework was determined to be " $5+3+X$ ". That is, 5 years of medical undergraduate education, 3 years of residential training and 1-4 years of specialty and subspecia;ty training (which equals to fellowship training in the U.S.). It is expected to develop the nationwide medical training system in 2020 , and lay foundation for construction of postgraduation medical education system [11].

In order to satisfy urgent needs for OAs, and to establish a replicable and standardized training system for OA specialists, Peking University Health Science Center (PKUHSC), one of the first ratified specialists training centers in China, initiated this explorative project. In 2017, PKUHSC formulated the "detailed rules for the current standardized training system of specialized anesthesiologists". The training duration is 3 years. The first 2 years were "generalized anesthesia training", followed by 1 year of subspecialty training (cardiothoracic anesthesia, pediatric anesthesia, obstetric anesthesia and advanced comprehensive anesthesia). Trainees could select only one subspecialty. OAs training program was launched in 2019 and was in continuous improvements.

PKUHSC has completed the training framework of OAs training project [12]. However, the standardized evaluation index has not been formulated. Therefore, with referencing to the subspecialty training objective/goals (see Supporting Information Appendix S1), we initiated a nation-wide investigation pooling opinions of experts from Youth Committee of China Anesthesiology Association (YCCAA) and formulated evaluation index for OAs using modified Delphi method.

\section{Methods}

\subsection{Construction of working group}

A working group was set up consisting of 8 members ( 1 director, 5 professors/associate professors, and 2 attending anesthesiologists). The initial survey instrument was devised, including the informed consent form, the instructions, and the questionnaire. The questionnaire consisted of 3 parts. The first part included surveys on the basic demographics of the experts. The second part surveyed the familiarity and 
evaluation standards of the experts. The third part is the drafted evaluation index, with three categories which further divided into 48 items (see Supporting Information Appendix S2).

\subsection{Experts panel selection}

We randomly distributed 40 questionnaires to experts (from 40 different hospitals in different regions of China) who should meet the following requirements: members of the YCCAA, with professional titles (professor/associate professor), having teaching experiences, having more than 10 years woking experiences in clinical anesthesia, and any two experts should not work in the same hospital. The process of generating the evaluation index and the overview of the procedure using Delphi method are described in detail in Fig. 1.

The preliminary evaluation index was assessed by the working group for clarity and completeness. Program directors were briefed on the concept of evaluation index and the purpose of the study prior to the initiation of the study, and was informed regularly with the progress. We agreed that at least 2 rounds of the Delphi surveys should be conducted to achieve a consensus among experts. The decision whether to undertake further rounds of the survey was depended on whether the results from the completed rounds represented a consensus. The questionnaire were distributed to experts by email, collected after predefined time interval of within one month, and were analyzed independently by two attending anesthesiologists in the working group who were blinded to the participation of the experts.

\subsection{First-Round of survey}

The first-round of the survey began on June 15th, and was completed on June 30th, 2020. Experts were asked for advice of the preliminary evaluation index, anonymously. In order to quantify the subjectivity of the evaluation, items were rated subsequently on a 4 - point Likert scale ( 1 = suggesting to delete the item, 2 = major revision of the item, $3=$ minor revision of the item, $4=$ agree with the item) [1]. Experts were also asked to provide any additional items they felt important and should be included in the evaluation index. Categories, sections and items that were rated by two or more experts as inappropriate, redundant, or incorrect were deleted or revised. In addition, experts were asked to rate the degree of familiarity of the field.

If only one expert deemed certain items as inappropriate, the working group would discuss and decide whether to delete or reserve these items. Further explanations of these decisions were included in the revised draft and distributed to experts for further comments in the subsequent rounds of the investigation. Additional suggestions made by experts were also incorporated anonymously in the modified evaluation index and distributed for further rounds of inquiry.

\subsection{Second-Round Survey}

The second-round survey was performed between July 25th, and August 25th, 2020. We send the revised questionnaire to experts who had completed the first round. Experts were also provided with the anonymous results from the first round with the suggestions of revised items. When no further 
suggestions or significant changes were brought forward, the results were compiled to create the final evaluation index.

\section{Data Analysis}

Data were collected and calculated for stability of the consensus, experts' enthusiasm, experts' authority, and experts' agreement [2].

\subsection{Stability of the consensus}

We evaluated the stability of consensus on the basis of the response consistency of the two rounds. In the present study, we considered the stability of consensus to be achieved if at least half of the experts agreed with the items, and there were minimal changes in the selections from the previous round.

\subsection{Experts' enthusiasm}

The experts' enthusiasm of performing the questionnaire were also evaluated. The enthusiasm coefficient $(\mathrm{E})$ is used to evaluate the enthusiasm of experts in participating the research. $\mathrm{E}=(\mathrm{Ec} / \mathrm{Et}) / 100 \%$. $\mathrm{Ec}$ indicates the number of experts who completed the questionnaire. Et is the total number of distributed questionnaire. Expert enthusiasm coefficient is expressed by the recovery rate of the consultation questionnaire, which mainly reflects the degree of experts' attention and involvement in the consultants to the current work. A higher value indicates the degree of attention and interest of the experts in completing the task. The maximum value is $100 \%$.

\subsection{Experts' authority}

The authority of experts (Au) is mainly evaluated by experts' self-evaluation, based on their familiarity with the consulting questions and the degree of judgement strength. Au is the mean value of the experts' academic level (Aac), the familiarity of the experts to the project (Afa), and the foundation of their judgement $(\mathrm{Afj}) . A u=(A a c+A f a+A f j) / 3$. The weight of experts' academic level were assigned according to their professional and academic ranks and titles (Table 1). Experts' familiarities (Table 2), and evaluation standard were also calculated (Table 3). 
Table 1

Weight of academic level

\begin{tabular}{|ll|}
\hline Academic classification & Weight \\
\hline Professorship and doctoral tutor & 1.0 \\
\hline Professorship and postgraduates supervisor & 0.9 \\
\hline Professorship but not as a tutor; & 0.7 \\
Associate professorship and postgraduates supervisor & \\
\hline Associate professorship & 0.5 \\
\hline Attending anesthesiologist & 0.3 \\
\hline
\end{tabular}

Table 2

Weight of experts' self-evaluated familiarity with the obstetric anesthesia

\begin{tabular}{|llll|}
\hline Strong & Medium & Weak & Not familiar \\
\hline 1.0 & 0.8 & 0.4 & 0 \\
\hline
\end{tabular}

Table 3

Experts' self-evaluation standard

\begin{tabular}{|llll|}
\hline & Degree & & Weak \\
\cline { 2 - 4 } & Strong & Medium & 0.3 \\
\hline Practical experience & 0.5 & 0.4 & 0.1 \\
\hline Theoretical analysis & 0.3 & 0.2 & 0.1 \\
\hline Peer experience & 0.1 & 0.1 & 0.1 \\
\hline Intuition & 0.1 & 0.1 & . \\
\hline Experts' were allowed to select only one item in the check-box for each four project. \\
\hline
\end{tabular}

\subsection{Experts' agreement}

Experts' agreement was compared between the two rounds with coefficient of variation ( $C v=$ standard deviation/averaged score). In addition, we calculated the Kendall's co-efficient of concordance $(W)$ by analyzing the quantified Likert scale to measure the agreement among experts' opinions [5].

\subsection{Statistics method}

All analyses were performed with SPSS 26 (SPSS Inc., Chicago, IL, USA). Descriptive statistics was performed to summarize the overall data. Kendall's coefficient concordance $W$ was calculated with nonparametric tests. 


\section{Results}

\subsection{Demographic information of the experts}

$39(97.5 \%)$ questionnaires were recovered in the first round. The demographic information of experts were shown in Table 4. Most of the experts were in $41-50$ years group $(n=24,62 \%)$. High academic authority was achieved in these experts, with 14 (36\%) associate professors, $25(64 \%)$ professors, and $28(72 \%)$ experts having doctorate degree. 7 (18\%) experts were doctoral tutors, and 27 (69\%) were post-graduate tutors. All 39 experts worked in grade-A tertiary hospitals with more than 10 years of working experiences. All experts were from hospitals of residency training base. Among these hospitals, 24 (62\%) were standardized training base for anesthesiology specialty, while 21 (54\%) hospitals have the intention of launching obstetric anesthesia training program. 
Table 4

Demographic information of the experts surveyed

Demographic information

frequency

(proportion \%)

Age(years)
$30 \sim 40$
$5(13 \%)$
$41 \sim 50$
$24(62 \%)$
$51 \sim$
$10(26 \%)$

Academic

title

\begin{tabular}{ll} 
Attending anesthesiology & 0 \\
\hline Associate professorship & $14(36 \%)$ \\
\hline Professorship & $25(64 \%)$
\end{tabular}

Highest academic degree

\begin{tabular}{ll} 
Undergraduate & $4(10 \%)$ \\
\hline Postgraduate & $7(18 \%)$ \\
\hline Doctorate & $28(72 \%)$
\end{tabular}

Hospital Classification

Tier and Level of the hospital

\begin{tabular}{ll}
$\begin{array}{l}\text { TRH } \\
\text { Grade-A }\end{array}$ & 39 (100\%) \\
\hline $\begin{array}{l}\text { TRH } \\
\text { Grade-B }\end{array}$ & 0 \\
\hline $\begin{array}{l}\text { TRH } \\
\text { Grade-A }\end{array}$ & 0 \\
\hline
\end{tabular}

\begin{tabular}{lll} 
Training base for residents & yes & $39(100 \%)$ \\
\cline { 2 - 3 } & no & 0 \\
\hline Training base for anesthesiology specialty & yes & $24(62 \%)$ \\
\cline { 2 - 3 } & no & $15(38 \%)$ \\
\hline $\begin{array}{l}\text { Planning to provide obstetric anesthesia training } \\
\text { programs }\end{array}$ & yes & $21(54 \%)$ \\
\cline { 2 - 3 } & no & $18(46 \%)$
\end{tabular}

Working

years

$\mathrm{TRH}$ : tertiary referral hospital 


\begin{tabular}{|ll|}
\hline Demographic information & $\begin{array}{l}\text { frequency } \\
\text { (proportion \%) }\end{array}$ \\
\hline $5 \sim 10$ & 0 \\
\hline $11 \sim 20$ & $15(38 \%)$ \\
\hline $21 \sim$ & $24(62 \%)$ \\
\hline Graduate students' tutor & $4(10 \%)$ \\
\hline Not a mentor & $27(69 \%)$ \\
\hline Postgraduates tutor (not as a Doctor tutor) & $7(18 \%)$ \\
\hline Doctor tutor & \\
\hline
\end{tabular}

TRH: tertiary referral hospital

\subsection{Stability of the consensus and the enthusiasm of the experts}

39 members from YCCAA responded and completed the first iteration of feedback, and 34 experts sent back their revision in the second round. It is generally accepted that a recovery rate of $50 \%$ is the minimum requirement for pooled analysis of expert opinion for stability [13]. Experts enthusiasm usually reflected by experts putting forward their opinions and the recovery rate of the questionnaire. The recovery rates of the two rounds were $97.5 \%$ and $87.2 \%$, respectively. Therefore, the stability of the consensus and experts enthusiasm in current survey were satisfiable.

\subsection{Experts' authority}

The authority of expert consultation is one of the key factors involved in the construction of the evaluation index. Based on previous research, an expert consultation authority coefficient $>0.7$ is considered to be reliable. An authority coefficient of 0.8 indicates even higher reliability of the experts' judgement $[2,6]$. In this study, the authority coefficient of the experts was $0.91 \pm 0.07$, which indicated that the experts' evaluation perspective are more experienced and reliable.

\subsection{Experts' agreement}

In the first round, the total number of selected items were " $3,42,11,1816$ " in correspondence to the Likert Scale of " $1,2,3$ and 4", respectively. In the second round, this reduced to 6 (Likert Scale of 3) and 1728 (Likert Scale of 4), respectively. When more than one experts raised different opinions for the same item, this one will be deleted, revised, or replaced. Likert scale was calculated to work out the $C v$ and the Kendall coefficient. In the first round, the $C v$ was $16.7 \%$; while in the second round, the $C v$ was $1.3 \%$. $C v$ within $15 \%$ is acknowledged as a reliable index for experts agreement [1]. The Kendall coefficient of the two rounds were $0.26(p=0.415)$ and $0.147(p=0.000)$, respectively, indicating consensus have been achieved by the experts in the second round of the inquiry. 


\subsection{Formulation of the evaluation index}

In the first round, major revisions were made in 11 items. 2 items were deleted (4-囚 "anesthesia management of maternal near miss." and 4- «"anesthesia management of E0-1 level immediate cesarean section"). 5 items were added (3-४" "perinatal anesthesia pharmacology"; $3-\bigotimes$, , "prevention and treatment of anesthesia complications reflux and aspiration, postpartum peripheral nerve injury, etc. "; 4- " "transferring of maternal and infants at high risk"; $5-\rrbracket$ "anesthesia for immediate and emergent cesarean section: high-

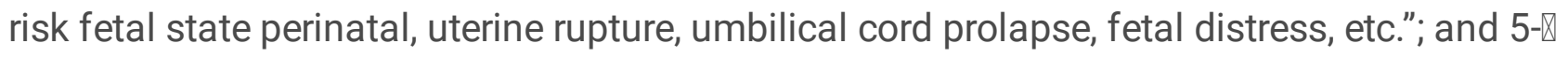

"management of acute laryngospasm, bronchospasm and asthma attack"). Major revisions were made

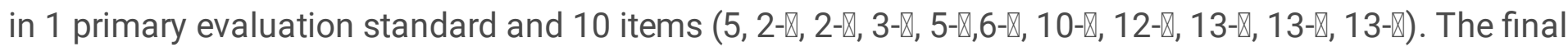
evaluation index was formulated and distributed to experts for further revision (see Supporting Information Appendix S3 and S4). In the second round of the survey, 34 (85\%) of the 40 experts completed the questionnaire. Only minor modifications and rephrases have been suggested. No significant changes after two rounds of the review indicated a convergence toward consensus. The final evaluation index consisted of 3 categories (practice ability, non-technical skills, and teaching and research capabilities) with 5 primary and 14 secondary evaluation standards which further divided into 51 items (Tables 5). 
Table 5

Evaluation Index for professional obstetric anesthesiologists

\section{Evaluation Primary and secondary evaluation standard with specific items Category}

\section{Practice ability}

A. Clinical thinking and decision-making

1.

Comprehensive analysis of clinical information
Including but not limited to:

$\triangle$ Fast access to clinical information (basic conditions, pregnancy related complications; ASA classification, functional status) and make anesthesia plans.

$\triangle$ Fetus and newborn assessment (interpretation of fetal heart rate monitoring, APGAR score).

$\triangle$ Mastering of evaluation methods (BLS, ABCDE, SAMPLE, 5H5T for emergent cases, etc.).

2. Decision making and implementation of clinical anesthesia
[ Selection of appropriate anesthesia method and anesthetic drugs according to pathophysiological characteristics of the pregnant women and surgical procedure.

[ Familiar with the pathophysiological changes to the pregnant women that may be caused by surgical operations.

冈lndividualized anesthesia for specific population.

B. Professionalism of obstetric anesthesia

BLS: basic life support.

$A B C D E: A$, airway; $B$, breathing; $C$, circulation; $D$, drug; $E, E C G$; and $F$, fibrillation.

SAMPLE: $S$, sign and symptom; $A$, allergy; $M$, medication; $P$, pertinent medical history; $L$, last meal or last menstrual period; $E$, event surrounding this incident.

5H5T: Hypokalemia/Hyperkalemia and other electrolytes; Hypothermia/hyperthermia; Hypovolemia; Hypoglycemia/Hyperglycemia; tablets; Tamponade; Thrombosis-pulmonary; Thrombosis-coronary; Tension-pneumothorax; asthma. 


\section{Evaluation Primary and secondary evaluation standard with specific items \\ Category}

3. Master the basic knowledge and skills of obstetric anesthesia
4 Perinatal anesthesia pharmacology

[ Pathophysiological changes during pregnancy

\ Physiological characteristics of fetus and newborns; Interpretation of the fetal heart monitoring.

$\nabla$ Indications and contraindications of anesthesia methods (intraspinal anesthesia, general anesthesia) for cesarean section; prevention and treatment of anesthesia complications.

\Labor analgesia, post-cesarean analgesia, etc.

$\otimes$ Anesthesia implementation of pregnant women undergoing nonobstetric surgery.

$\nabla$ Prevention and treatment of anesthesia complications (reflux and aspiration, postpartum peripheral nerve injury, etc.) .

4. Anesthesia for high risk pregnant women
प Anesthesia for pregnant women with non-obstetric diseases at high-risk : cardiovascular system (congenital heart disease, pulmonary hypertension, aortic aneurysm, cardiomyopathy, heart failure, etc.), respiratory system, endocrine system, hematology, liver and kidney dysfunction, mental illness, nervous system diseases, infectious diseases, multi-drug abuse, severe obesity, septic shock, etc.

૫ Transferring of maternal and infants at high risk.

BLS: basic life support.

$A B C D E: A$, airway; $B$, breathing; $C$, circulation; $D$, drug; $E$, ECG; and F, fibrillation.

SAMPLE: S, sign and symptom; A, allergy; $M$, medication; P, pertinent medical history; L, last meal or last menstrual period; $E$, event surrounding this incident.

5H5T: Hypokalemia/Hyperkalemia and other electrolytes; Hypothermia/hyperthermia; Hypovolemia; Hypoglycemia/Hyperglycemia; tablets; Tamponade; Thrombosis-pulmonary; Thrombosis-coronary; Tension-pneumothorax; asthma. 


\section{Evaluation Primary and secondary evaluation standard with specific items \\ Category}
5. Anesthesia for obstetrics needing immediate treatment in the OR and in pre- hospital emergency.
4. Anesthesia for immediate and emergent cesarean section (high- risk fetal state perinatal, uterine rupture, umbilical cord prolapse, fetal distress, etc.)
(] Obstetric hemorrhage
( Respiratory and cardiac arrest of maternal and the newborn
$\triangle$ Amniotic fluid embolism, pulmonary embolism, air embolism
$\unrhd$ Acute heart failure
\Anaphylactic shock
$\otimes$ Local anesthetics intoxication
$\nabla$ Obstetric difficult airway
\ Management of acute laryngospasm, bronchospasm and asthma attack.
6. Mastering and application
4 Transesophageal/thoracic ultrasound application in obstetrics; of advanced Hemodynamic monitoring with advanced apparatus (recommended anesthesia technique for senior obstetric anesthesiologists).
4 Ultrasound guided regional nerve block and central venous catheterization for obstetrics.
Q Thromboelastogram; Standardized blood transfusions in Obstetrics; Autologous blood transfusion in obstetrics, etc.

Non-technical skills

C. Comprehensive skills

7. Teamwork $\quad$ P Possessing decision-making leadership; providing constructive medical opinion.

4. Multidisciplinary teamwork cooperation.

$\nabla$ Realizing the potential of team members.

BLS: basic life support.

$A B C D E$ : $A$, airway; $B$, breathing; $C$, circulation; $D$, drug; $E, E C G$; and F, fibrillation.

SAMPLE: S, sign and symptom; A, allergy; $M$, medication; $P$, pertinent medical history; $L$, last meal or last menstrual period; $E$, event surrounding this incident.

5H5T: Hypokalemia/Hyperkalemia and other electrolytes; Hypothermia/hyperthermia; Hypovolemia; Hypoglycemia/Hyperglycemia; tablets; Tamponade; Thrombosis-pulmonary; Thrombosis-coronary; Tension-pneumothorax; asthma. 


\section{Evaluation Primary and secondary evaluation standard with specific items Category}

8. Communication sills

9.

Professionalism
[ Using verbal and nonverbal means to communicate effectively with patients, family members, colleagues and the public.

[ Ability to coordinate and utilize public health resources, providing necessary medical-related guidance and services.

4. Possess the professional spirit of assuming professional responsibilities and willingness of following ethical principles.

4 Ability to continuously improve the quality of clinical anesthesia.

$\nabla$ Stress-management, self-adjustment to work pressure.

\Compliance to medical laws and regulations.

\section{Teaching and research capabilities}

D. Learning and teaching ability

10. Independent $\square$ Completion of periodic specialized training regular assessment. learning ability

प Fluency in professional English.

$\nabla$ Certification for simulation evaluation.

11. Teaching $\square$ Clinical teaching. ability

[ Instruct residents to conduct clinical case analysis.

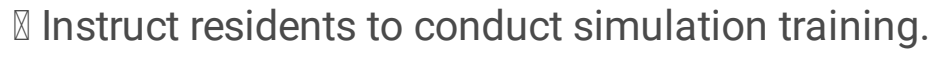

E. Research ability

12. Innovation $\quad \square$ Ability to raise and solve clinical problems.

प Medical related innovation.

\Have a knowledge of the application, recognition, and transformation of intellectual property.

BLS: basic life support.

$A B C D E: A$, airway; $B$, breathing; $C$, circulation; $D$, drug; $E, E C G$; and F, fibrillation.

SAMPLE: S, sign and symptom; A, allergy; $M$, medication; P, pertinent medical history; $L$, last meal or last menstrual period; $E$, event surrounding this incident.

5H5T: Hypokalemia/Hyperkalemia and other electrolytes; Hypothermia/hyperthermia; Hypovolemia; Hypoglycemia/Hyperglycemia; tablets; Tamponade; Thrombosis-pulmonary; Thrombosis-coronary; Tension-pneumothorax; asthma. 


\section{Evaluation Primary and secondary evaluation standard with specific items Category}
13. Research ability
प Mastering of basic scientific theories and experimental skills for fundamental research.
4 Knowing how to performing clinical research.
Q Performing research using interdisciplinary platform.
ه Capable of research design, implementation, and thesis writing.
14. Research integrity
प Follow the requirements of scientific research integrity.
[ Conduct research in compliance with the ethical requirements.

BLS: basic life support.

$A B C D E: A$, airway; $B$, breathing; $C$, circulation; $D$, drug; $E, E C G$; and F, fibrillation.

SAMPLE: S, sign and symptom; A, allergy; $M$, medication; P, pertinent medical history; L, last meal or last menstrual period; $E$, event surrounding this incident.

5H5T: Hypokalemia/Hyperkalemia and other electrolytes; Hypothermia/hyperthermia; Hypovolemia; Hypoglycemia/Hyperglycemia; tablets; Tamponade; Thrombosis-pulmonary; Thrombosis-coronary; Tension-pneumothorax; asthma.

\section{Discussion}

Obstetric anesthesia is a subspecialty of anesthesia dedicated to peripartum, perioperative, pain and anesthetic management of women during pregnancy and the puerperium. The unique skills of OAs in emergency resuscitation and critical illness management make them of special importance in perinatal management in high-risk patients [14]. PKUHSC is pioneered in initiating the anesthesia specialty and subspecialty training program in 2019. Its training objectives, duration, specialty curriculums, technical skilles were drafted and approved by Post Graduation Continuing Education Committee of PKUHSC under continuous improvement. In 2021, the draft of the "detailed rules for the current standardized training system of specialized anesthesiologists" was revised to include more specific regulations. However, the standardized evaluation index was not formulated till now. In the current study, we have drafted the initial evaluation index with reference to OA training objectives, and solicited opinions from YCCAA experts extensively. We hope this evaluation index could be adopted as a reference to help establish standardized assessment tool by other institutions.

\subsection{Experts selection}

In the current study, the Delphi method is adopted as a structured process to collect opinions from experts by questionnaires distributed with controlled opinion feedback [15]. Considering experts from different regions may have difficulty in achieving consensus on the nuances, we made an initial draft based on the 
training objectives of OA subspecialty of PKUHSC. In order to guarantee acquiring comprehensive, reliable and expertise advice, we distributed questionnaires to experts in YCCAA.

Most of the experts were relatively young, holding high academic ( $72 \%$ with doctorate degree) and professional (36\% of associate professor and $64 \%$ professor) authority in tertiary referral hospitals nationwide, and excelled in clinical practice, medical education and research, and holding concurrent posts as director/deputy director of anesthesiology department, respectively. They witnessed the rapid development of anesthesiology in China in recent decade, and could provide valuable professional insights of medical education in macro-perspective.

\subsection{Analysis of the revisions in the evaluation index}

Analysis of the revised items in the evaluation index revealed that experts' interests were focused on the clinical "practice ability" (including 2 primary evaluation standards which further divided to six secondary evaluation standards and 27 items). Among these, 2 items (4- $\Downarrow$ and $4-\bigotimes)$ were deleted. 5 new items (3- $\, 3-$

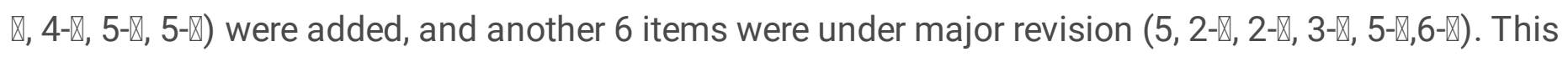
accounted for $72 \%(13 / 18)$ of all revised ones, and $48 \%$ of all "practice ability" items. With the development of ultrasound guided nerve-block technique in obstetric patients and the increased number of cesarean section under general anesthesia, corresponding assessment requirements were added or revised.

No changes were made in the non-technical skills. We speculated that this might be attributed to the difficulty of making objective, direct and accurate assessment for trainees concerning the ability of "teamwork, communication skills, and professionalism". The working scenario simulation teaching method and other corresponding teaching strategies were not widely implemented in training hospitals in China. More innovative reforms were to be performed in this field. It was noticeable that $28 \%$ revisions $(10-\nabla, 12-\rrbracket, 13-\rrbracket, 13-\rrbracket, 13-\nabla)$ were made in the category of "teaching and research capabilities". This accounted for $33 \%$ items of the this category. More attentions have been paid to the research ability, and 4 items (44\%) were suggested to be revised.

\subsection{Assessment of research ability}

With more emphasis on the research ability for fellowship training worldwide, some advocated extending the training duration exclusively for scientific research. While other analysis believed that the scientific output were not increased with proportion to the extended training time, and this might deter the enthusiasm of potential trainees [16]. Teaching and research capabilities, as one of major categories, was also included in our evaluation index. It was intended to have trainees possess basic scientific research skills, and to lay foundation for them to become clinician-scientists and participate in research programs after years of accumulation in their professional field. This is also one of the future development goals of research-oriented hospitals.

\subsection{Future challenges}


The implementation of this pilot program is a gradual process. In 2012, the Accreditation Council for Graduate Medical Education (ACGME) officially recognized and accredited Obstetric Anesthesiology Fellowship. In 2015, the American College of Obstetricians and Gynecologists (ACOG) and the Society for Maternal Fetal Medicine (SMFM) issued the consensus document for improved referral and regionalization of high-risk obstetric services (antepartum through postpartum care), which stipulated that the maternal care centers should be equipped with staffings of OBs. Level II (specialty care) need a "board-certified anesthesiologist with special training or experience in obstetrics, available for consultation," and for levels III (subspecialty care) and IV (regional perinatal health care centers), a "board-certified anesthesiologist with special training or experience in obstetrics is in charge of obstetric anesthesia services." [17].

While in China, there is no such organizations or regulations. During the alternating period of the old and new specialists training system, anesthesilogists finishing "generalized anesthesia training" were permitted in obstetric anesthesia in comprehensive hospitals. Moreover, obstetric anesthesia is a subspecialty which has a higher possibility of being involved in legal suits of adverse neonatal outcomes, and the work load of OA in China were heavy. In addition, the capacity of OA training in PKUHSC teaching hospitals were limited, with only 1-2 positions every year. Considering the training duration, practicing scopes and risks, as well as the supporting incentive policies for career prospects, there was seldom a trainee would select OA as his/her career priority for the moment. More arduous work were to be done to explore how to improve the incentives to attract more trainees to this subspecialty along with establishment and improvement of OA training program. Moreover, maintenance of certification to guarantee the quality of competency was not implemented in all specialty and subspecialty in China. This periodic accreditation for comprehensive evaluation is expected in the future [18]. The enrolled OA trainees were expected to finish the program at the end of the year 2021.

\section{Conclusion}

The objective of OA training not only include anesthetic management of parturient at high-risk, but also time -sensitive medical management of critically ill parturients and neonates, and to help graduates become "peri-delivery physicians" [19]. This study used modified Delphi method to reach a consensus on the evaluation standard of obstetric anesthesiologists. After two rounds of the survey, the final evaluation index consisted of 3 categories (practice ability, non-technical skills, and teaching and research capabilities) with 5 primary and 14 secondary evaluation standards which were further divided into 51 items. More attentions were foucused on clinical practice ability and research training. We hope this pilot program could be continuously improved, and help establish a replicable and popularized standardized evaluation index for obstetric anesthesiologists training.

\section{Declarations}

\section{Availability of data and material}


All data generated or analyzed during this study are included in this published article. More detailed information could be found in Supporting Information Appendix S1-S3.

\section{Consent for publication:}

All authors consent the publication of the this article.

\section{Funding}

The present study was supported by the project of National Steering Committee for Postgraduate Education of Medical Professional Degree (B3-YX20190302-03) and the Educational and Teaching Research Project of Peking University Health Science Center (2019YB32). These funding support the design, implementation and publication of the current study.

\section{Authors' contributions}

Guarantor of integrity of entire study: $X Z, Y Z, H Z, J W, M L, C W, X G, M X$.

Literature research: $\mathrm{XZ}, \mathrm{YZ}, \mathrm{HZ}, \mathrm{CW}$.

Formulation of the initiation evaluation index: $X Z, Y Z, H Z, J W, M L, C W$.

Data analysis: $X Z, Y Z, H Z, C W$.

Manuscript editing and revision: $X Z, Y Z, H Z, X G, M X$.

Manuscript final version approval: JW, ML, XG, MX.

all authors have read and approved the manuscript.

\section{Acknowledgments}

None.

\section{Competing interests}

The authors declare no competing interests.

\section{Ethics approval and consent to participate}

Not applicable.

\section{References}

1. Wisman-Zwarter N, van der Schaaf M, Ten Cate O, Jonker G, van Klei WA, Hoff RG. Transforming the learning outcomes of anaesthesiology training into entrustable professional activities: A Delphi 
study. Eur J Anaesthesio, 201,33(8):559-567. DOI: 10.1097/EJA.0000000000000474.

2. Dai F, Wei K, Chen Y, Ju M. Construction of an index system for qualitative evaluation of undergraduate nursing students innovative ability: A Delphi study. J Clin Nurs, 2019,28(23-24):43794388. DOI: 10.1111/jocn.15020. Epub 2019 Oct 1.

3. Weiniger CF. What's new in obstetric anesthesia in 2018? Int J Obstet Anesth. 2020 May,42:99-108. doi: 10.1016/j.ijoa.2020.03.002. Epub 2020 Mar 19. PMID: 32278531.

4. Liang J, Li X, Kang C, Wang Y, Kulikoff XR, Coates MM, Ng M, Luo S, Mu Y, Wang X, Zhou R, Liu X, Zhang Y, Zhou Y, Zhou M, Li Q, Liu Z, Dai L, Li M, Zhang Y, Deng K, Zeng X, Deng C, Yi L, Zhu J, Murray CJL, Wang H. Maternal mortality ratios in 2852 Chinese Counties, 1996- 2015: and achievement of Millennium Development Goal 5 in China: a subnational analysis of the GIOAI Burden of Disease Study 2016. Lancet, 2019,393:241-252. DOI: 10.1016/S0140-6736(18) 31712-4. Epub 2018 Dec 13

5. Wu Y, Jin A, Xie G, Wang L, Liu K, Jia G, Liang X, Xu J. The 20 Most Important and Most Preventable Health Problems of China: A Delphi Consultation of Chinese Experts. Am J Public Health. 2018 Dec,108(12):1592-1598. doi: 10.2105/AJPH.2018.304684. Epub 2018 Oct 25. PMID: 30359111, PMCID: PMC6236763.

6. Long Y, Zhou X, Deng P, Liao X, Wu L, Zhou J, Huang H. Construction of a physiological aging scale for healthy people based on a modified Delphi method. Zhong Nan Da Xue Xue Bao Yi Xue Ban, 2016,1(4):422-426. DOI: 10.11817/j.issn.1672-7347.2016.04.014.

7. Di GH, Yao SL, Wang J, Wu ZL, Liu H, Wang H. Factors influencing the improvement of neuraxial labor analgesia in China: a questionnaire survey. Chin Med J 2020,133:613-614. doi: 10.1097/CM9. 0000000000000529.

8. Wu J, Yao SL. Obstetric anesthesia in China: associated challenges and long-term goals. Chin Med J (Engl). 2020 Mar 5,133(5):505-508. doi: 10.1097/CM9.0000000000000664. PMID: 32142490, PMCID: PMC7065864.

9. Souza JP, Gülmezoglu A, Lumbiganon P, Laopaiboon M, Carroli G, Fawole B, Ruyan P, WHO GIOAI Survey on Maternal and Perinatal Health Research Group. Caesarean section without medical indications is associated with an increased risk of adverse short-term maternal outcomes: the 20042008 WHO GIOAI Survey on Maternal and Perinatal Health. BMC Med, 2010,8:71. DOI: 10.1186/17417015-8-71.

10. Birnbach DJ, Bateman BT. Obstetric Anesthesia: Leading the Way in Patient Safety. Obstet Gynecol Clin North Am. 2019 Jun,46(2):329-337. doi: 10.1016/j.ogc.2019.01.015. PMID: 31056134.

11. Haiying Tang, Jia Liu, Jian Bl, etc. Evaluation of the Development and Challenges of Chinese Specialist Training from the Differences between Chinese and American Medical Education, 2019, 027(004):541-544.

12. Ying Yang, Liyun Zheng, Ruoning Wang, etc. Exploration on building up standardized fellowship training system. Chin J Med Edu. 2020, 40(06):471-475. 
13. Keeney S, Hasson F, McKenna $\mathrm{H}$. Consulting the oracle: ten lessons from using the Delphi technique in nursing research. J Adv Nurs, 2006,53(2):205-212. DOI: 10.1111/j.1365-2648.2006.03716.x.

14. Kuczkowski KM. Running an obstetric anesthesia training program: words of wisdom. Arch Gynecol Obstet. 2009 Dec,280(6):883-8. doi: 10.1007/s00404-009-1069-1. Epub 2009 Apr 9. PMID: 19357863.

15. Chin KJ, Tse C, Chan V, Tan JS, Lupu CM, Hayter M. Hand motion analysis using the imperial college surgical assessment device: validation of a novel and objective performance measure in ultrasoundguided peripheral nerve blockade. Reg Anesth Pain Med, 2011,36(3):213-219. DOI:

10.1097/AAP.0b013e31820d4305

16. Kacmar RM, De Oliveira GS Jr, McCarthy RJ, Wong CA. Status of obstetric anesthesiology fellowship research education in the USA and Canada: a 2013 survey of fellowship program directors. Int $J$ Obstet Anesth. 2015 May,24(2):193-4. doi: 10.1016/j.ijoa.2014.11.005. Epub 2014 Nov 28. PMID: 25554674.

17. Gelber K, Kahwajian H, Geller AW, Zakowski MI. Obstetric Anesthesiology in the United States: Current and Future Demand for Fellowship-Trained Subspecialists. Anesth Analg. 2018 Dec,127(6):14451447. doi: 10.1213/ANE.0000000000003809. PMID: 30252705.

18. Miller SH. American Board of Medical Specialties and repositioning for excellence in lifelong learning: maintenance of certification. J Contin Educ Health Prof. 2005 Summer,25(3):151-6. doi: 10.1002/chp.22. PMID: 16173049.

19. Bateman BT, Tsen LC. Anesthesiologist as epidemiologist: insights from registry studies of obstetric anesthesia-related complications. Anesthesiology. 2014,120:1311-1312

\section{Figures}


Construction of the working group \& Inclusion and exclusion criteria of experts $\rightarrow$ Preliminary draft of evaluation index

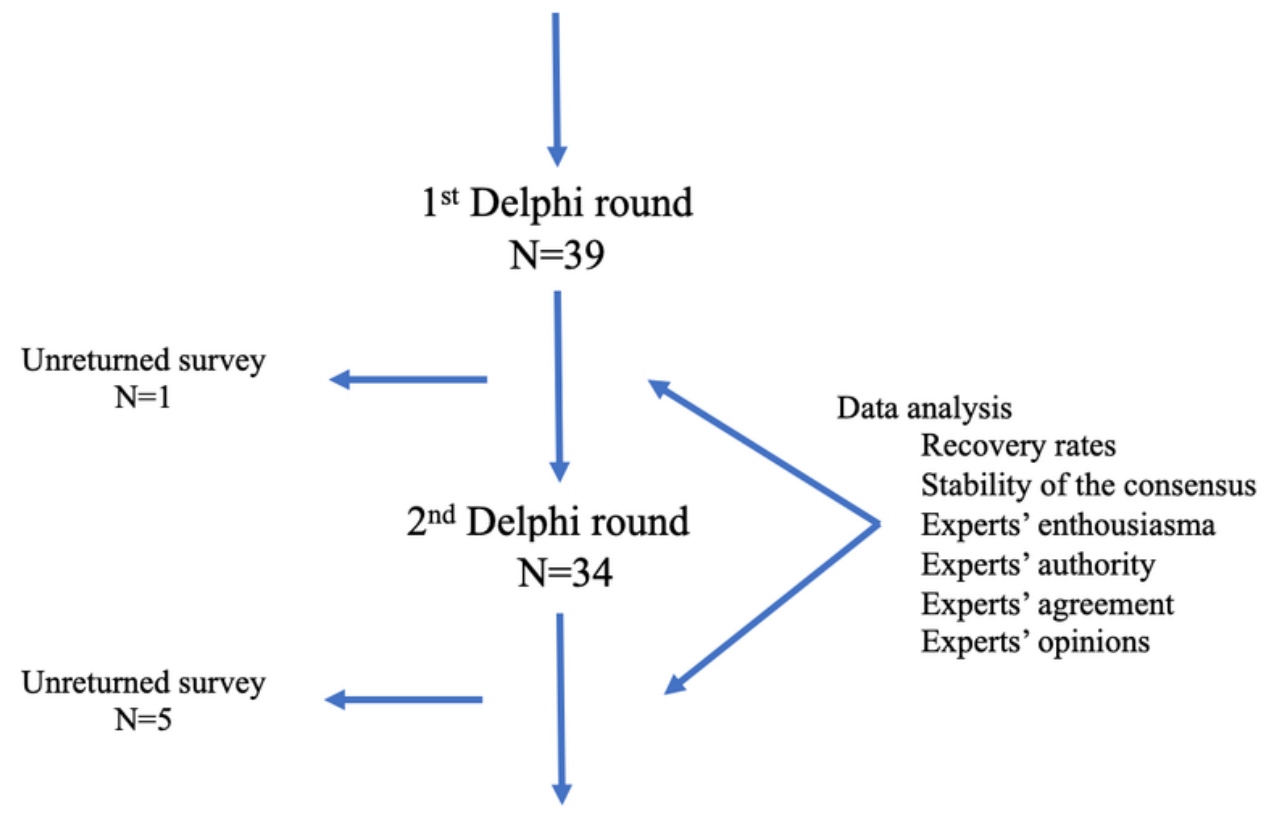

Final evaluation index for OBA training

\section{Figure 1}

Process of generating the list of evaluation index and the overview of the procedure using Delphi method. $\mathrm{OA}$, obstetric anesthesiologists.

\section{Supplementary Files}

This is a list of supplementary files associated with this preprint. Click to download.

- 8.15supportinginformation.docx 